\title{
MOTIVACÕES PARA SER (IN)ATIVO EM PERÍODO PANDÉMICO: UM ESTUDO ÉXPLORATÓRIO COM ADOLESCENTES E JOVENS ADULT@S
}

\author{
Isabel Condessa \\ Faculdade de Ciências Sociais e Humanas da Universidade dos Açores (FCSH-UA) \\ CIEC, Instituto de Educação da Universidade do Minho, Braga, Portugal \\ maria.id.condessa@uac.pt \\ Zélia Caçador Anastácio \\ CIEC, Instituto de Educação da UUniversidade do Minho, Braga, Portugal \\ zeliaf@ie.uminho.pt \\ Celeste Antão \\ Instituto Politécnico de Bragança, Escola Superior de Saúde do IPB, Health Sciences Research Unit: Nursing \\ (UICISA) \\ celeste@ipb.pt
}

Recepción Artículo: 27 octubre 2021 Admisión Evaluación: 27 octubre 2021 Informe Evaluador 1: 28 octubre 2021 Informe Evaluador 2: 29 octubre 2021

Aprobación Publicación: 30 octubre 2021

\section{RESUMO}

Com este estudo exploratório pretendemos demarcar a influência de diversas motivações para a adesão à prática de atividade física (AF), num grupo de adolescentes e jovens adultos/as ( $n=186$ ) em 2020, aquando dos períodos de confinamento pandémico, imposto pela COVID-19. Neste sentido, construímos um questionário no Google Forms, o qual foi aplicado on-line e nos permitiu recolher testemunhos sobre algumas questões: características individuais - dados biográficos (idade, sexo, índice de massa corporal - IMC, habilitações académicas, ocupação, alterações gerais de peso desde o primeiro confinamento) e motivos de maior ou menor adesão a uma prática regular de atividade física. Foi realizada uma análise dos dados com o programa SPSS (versão 27.0) e recorremos a provas estatísticas variadas para análise descritiva (frequência; percentagem), comparativa [média e desvio padrão; Qui Quadrado $\left(X^{2}\right)$ ] e correlacional [Spearman ( )]. Os resultados permitem-nos concluir que os inquiridos do estudo, alguns já em situação de risco quanto IMC, sofreram maiores alterações de peso, situação que registou valores diferentes consoante 0 sexo, 0 IMC e a ocupação naquele momento. 0 nível de adesão à pratica de AF regular oscilou, havendo um número aproximado dos que tiveram maior e menor envolvimento em práticas e, numa maior quantidade de tempo passada em casa, liderou 0 trabalho de AF seguindo tutoriais e orientação por profissionais, nomeadamente de clubes e de educação física. Os motivos aduzidos a um maior nível de prática de AF regular e os mais favoráveis a essa prática foram: i) 0 gosto pela atividade física e com reforço positivo para as atividades de lazer ao ar livre e negativo para as práticas de ginásio; ii) a melhoria das 


\section{MOTIVAC̄̃ES PARA SER (IN)ATIVO EM PERÍOdO PANDÉMICO: UM ESTUdO EXPLORATÓRIO COM ADOLESCENTES E JOVENS ADULT@S}

capacidades físicas e a saúde e o bem-estar. As motivações de estética tiveram menos relevância e, quanto à importância do cuidado pelo corpo, foi o controlo da massa corporal que prevaleceu.

Palavras-chave: pandemia COVID-19; adolescentes e jovens adultos; atividade física; motivos de adesão

\section{ABSTRACT}

Motivations to be (in)active in a pandemic period: an exploratory study with adolescents and young adults. With this exploratory study we intend to demarcate the influence of various motivations for adherence to physical activity (PA) in a group of adolescents and young adults $(n=186)$ in 2020, during the periods of pandemic lockdown imposed by COVID-19. In this sense, we built a questionnaire on Google Forms, which was applied online and allowed us to collect testimonies on some issues: individual characteristics biographical data (age, gender, body mass index - BMI, academic qualifications, occupation, general changes in weight since the first lockdown) and reasons for greater or lesser adherence to a regular practice of physical activity. Data analysis was performed using SPSS software (version 27.0) and we used several statistical tests for descriptive (frequency; percentage), comparative [mean and standard deviation; Chi Square (X2)] and correlational [Spearman ( )] analysis. Our results allow us to conclude that the respondents of the study, some already at risk with regard to BMI, suffered greater changes in weight, a situation that registered different values according to gender, BMI and occupation at that moment. The level of adherence to regular PA practice fluctuated, with an approximate number of those who had more and less involvement in practices and, in a greater amount of time spent at home, led PA work following tutorials and guidance by professionals, namely from clubs and physical education. The reasons adduced to a higher level of regular PA practice and the most favourable to this practice were: i) the enjoyment of physical activity and with positive reinforcement to outdoor leisure activities and negative reinforcement to gym practices; ii) the improvement of physical abilities and health and well-being. Aesthetic motivations had less relevance and, regarding the importance of body care, it was the control of body mass that prevailed.

Keywords: pandemic COVID-19; adolescents and young adults; physical activity; reasons for adherence

\section{INTRODUÇÃO}

Em 2020 as nossas vidas foram transformadas com a nova crise mundial que adveio do aparecimento da doença COVID-19 e o formato de vida socialmente aceite sofreu com mudanças de comportamentos, inclusive de crianças, adolescentes e jovens adultos/as. Se até à data tínhamos preocupações com o elevado sedentarismo desta população e, consequentemente com as comorbilidades associadas, sendo já preocupação do Sistema Nacional de Saúde (SNS) português divulgar programas de promoção de práticas de Atividade Física regular e moderada ou intensa, agora as medidas de confinamentos sucessivos, as preocupações com os direitos humanos e com o bem-estar passou para um segundo plano, e a prevenção da falta de movimento foi ultrapassada por outras medidas mais urgentes de prevenção ao contágio da COVID-19, onde o isolamento físico e, consequentemente o social, foi a única medida com impacto positivo para o tratamento e prevenção imediata da propagação do vírus SARS-CoV-2.

A pandemia foi decretada pela Organização Mundial de Saúde (OMS) em 21 de março de 2020, tendo esta organização criado em abril desse mesmo ano a campanha BeAtive (WHO, 2020), na medida em que vários estudos salientam a importância da prática de atividade física regular, mesmo durante a pandemia, pois esta prática cria "melhor função imunitária e defesas do organismo contra agentes infeciosos" (Almeida, et al., 2021, p. 36). Também para estes autores, a Atividade Física é considerada como principal estratégia preventiva e não-farmacológica para a prevenção de variadas doenças, desde as metabólicas, às fisiológicas, físicas, psicológicas e sociais.

As mudanças de comportamentos, requeridas pelas medidas preventivas e no limite, no confinamento, resultaram no distanciamento físico e social, assim como no dispêndio excessivo de tempo de exposição a écrans em casa, usados quer para teletrabalho, quer para ensino a distância, acrescendo aos momentos de entretenimento 
já estabelecidos. A estas rotinas associou-se a redução de movimento e, na globalidade, fruto deste sedentarismo acrescido, deparamo-nos com: a) alterações de peso associadas ao aumento da massa adiposa e/ou redução da massa muscular; b) comorbilidades várias, em que para além da obesidade, foram se registando problemas de aumento de açúcar, hipertensão e transtornos psicossociais e relacionados com a autoestima.

A World Health Organization (WHO, 2018) antes do período pandémico propunha que fossem criadas condições para que existissem ambientes promotores de atividade física nos locais onde as pessoas viviam e trabaIhavam, contribuindo para o bem-estar humano, incluindo a saúde física e mental. Esta instituição organizativa, assim como vários autores, vão alertando para a importância de os programas de atividade física nas escolas contribuírem para desenvolver a aptidão física e alfabetização em saúde, habilidades motoras e atitudes e hábitos de vida positivos e fazer aumentar o prazer geral pela atividade física. Contudo, registamos ainda que muitos jovens, embora sujeitos a um período escolar de cerca de 12 anos com Atividade Física curricular, nem sempre adquirem uma atitude positiva ou uma motivação acrescida para a adesão a uma prática regular de exercício físico, conforme advogam vários autores (Condessa, 2015; Seabra, 2017).

\section{OBJETIVOS DA INVESTIGAÇÃO}

Esta investigação teve como objetivo analisar a influência de diversos parâmetros na motivação e adesão à prática de atividade física AF, em período pandémico, de um grupo de adolescentes e jovens adultos/as provenientes de uma mesma região autónoma de Portugal - Açores.

\section{AMOSTRA E PARTICIPANTES}

A amostra deste nosso estudo foi constituída por 186 indivíduos do Arquipélago do Açores, adolescentes (com 86,5\% de idade até 25 anos e 30,6\% até 18 anos) e jovens adultos/as, predominantemente do sexo feminino $(72,6 \%)$, estudantes ou jovens em início de carreira profissional, que foram selecionados de forma aleatória simples.

\section{METODOLOGIA E INSTRUMENTO DE RECOLHA DE DADOS}

Este estudo, seguiu uma metodologia quantitativa e foi do tipo transversal, tendo os sujeitos da amostra preenchido 0 instrumento de recolha de dados num único momento (Cohen, Manion \& Morrison, 2017). Carateriza-se por ser também um estudo descritivo e correlacional dado o tipo de análise de dados efetuado com vista a atingir os objetivos definidos. Para o propósito desta investigação, construiu-se um questionário com base noutros já anteriormente usados pelas autoras (Condessa \& Anastácio, 2019; Anastácio, 2016). 0 estudo foi realizado com base numa recolha dados efetuada em 2020, foi desenvolvido a partir da aplicação do questionário aleatoriamente através do Google Forms, o qual antevia logo de início uma questão de consentimento informado sem a qual não era permitido avançar, cumprindo-se assim os procedimentos eticamente previstos. 0 questionário era subordinado ao tema "Conhecer estilos de vida e comportamentos de adolescentes e jovens adultos com impacto no seu Bem-estar no Período de Pandemia COVID-19 no ano de 2020". Era um questionário misto, pois tinha umas questões abertas e outras fechadas de escolha múltipla. Como questões de escolha múltipla tínhamos a Análise de Fatores de Adesão e de Motivação para a prática de AF, referentes a variáveis dependentes, com os seguintes scores para 0 nível de importância: 1 = nada importante; 2 = pouco importante; $3=$ importante; 4 = muito importante. Nas questões abertas, as respostas eram curtas ou de inclusão de dados biométricos, nomeadamente idade, peso(s) e altura, consideradas variáveis independentes ou fatores. Na sua análise procuramos verificar a influência de algumas variáveis independentes na adesão à prática de uma Atividade Física Regular e nas motivações para a mesma prática. Neste sentido elegemos as características pessoais (biodados), com realce para e mudança física (alteração de peso); características psicológicas e de atitude (motivação para a prática de AF e adesão à AF). Para efeitos de análise e tratamento estatístico dos dados recolhidos utilizou-se 0 software IBM Statistical Package for the Social Sciences (SPSS, versão 27.0). Na elaboração da base de dados procedeu-se à categorização das variáveis: idade (adolescentes; jovens adultos); habilitação académica; ocupa- 
ção; grupo de índice de massa corporal (IMC); e mudança de peso em 2020. Recorremos a provas estatísticas variadas para análise descritiva e comparativa [média e desvio padrão; frequência e percentagens; Qui Quadrado de Pearson ( $\left.\mathrm{X}^{2}\right)$ ] e correlacional não paramétrica [Ró de Spearman ( )].

\section{RESULTADOS}

Para apresentação dos resultados partimos de uma caracterização da amostra (Tabela 1), das suas perceções de adesão à AF ocorrida em contexto de pandemia no ano de 2020 (Figura 1) e das motivações que lhe estão associadas (Tabela 2)

Na análise das características sociodemográficas (Tabela 1) constatámos que a nossa amostra tinha uma média de idade de 20.5 anos (variação de 4.15 anos), apresentando uma distribuição diferenciada em relação a caraterísticas como o sexo, em que predominaram as raparigas/mulheres (72.6\%), habilitação académica e ocupação, sendo a maioria estudantes (78.0\%), sobretudo do ensino secundário e profissional (61.3\%).

Fisicamente, é de realçar que quanto ao índice de massa corporal (IMC) havia prevalência de adolescentes e jovens adultos de peso normal (68.3\%) realçando-se aqui ainda um elevado número daqueles que apresentavam excesso de peso ou obesidade (26.3\%).

Relativamente a alterações de peso no período de confinamento, um terço não sofreu alterações (33.3\%). Contudo, a maior alteração registou-se em aumento de peso (41.8\%), enquanto a diminuição foi registada por apenas $18.8 \%$.

Tabela 1 - Caraterização da amostra estudada -

Dados biométricos e Sociodemográficos de 2020 (Fonte: Autoras)

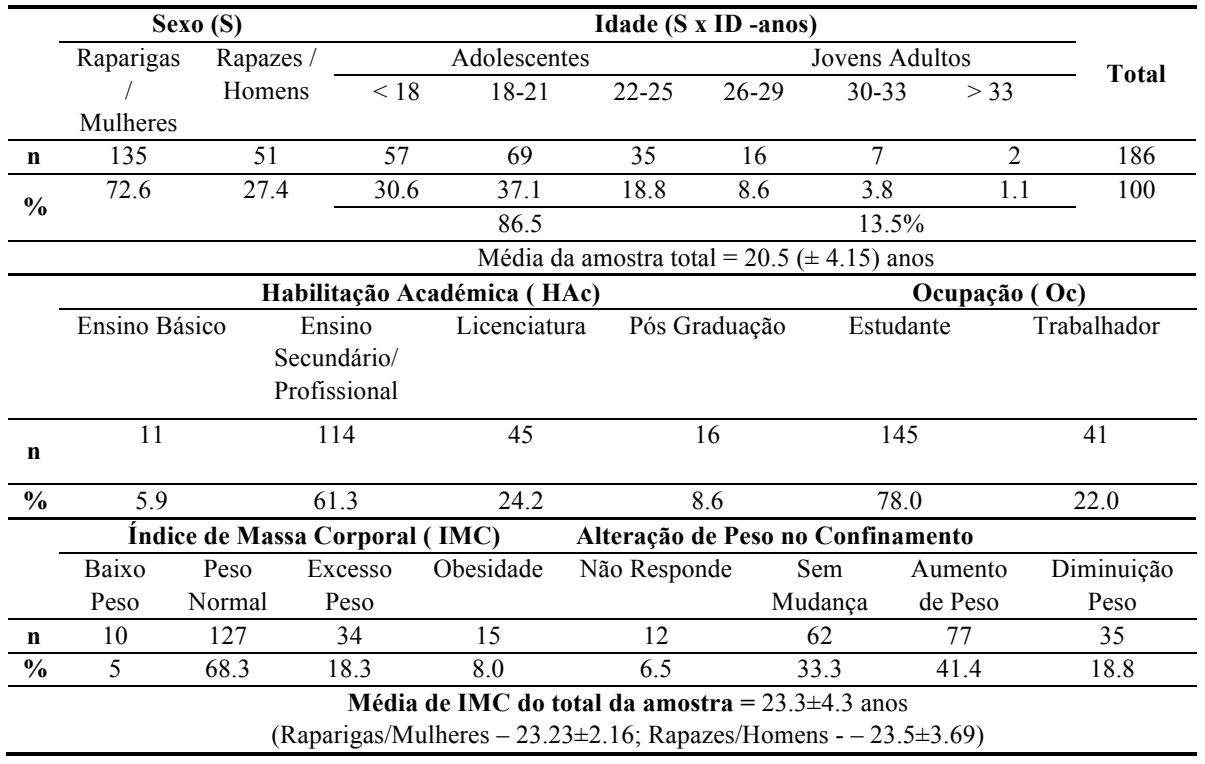

Encontramos diferenças significativas para a variável "mudança de peso em 2020" entre os adolescentes e jovens adultos. Como podemos verificar, dos jovens em estudo muitos afirmaram ter sofrido alterações de peso durante os períodos de confinamento (aumento para cerca de $42 \%$ e diminuição para cerca de $20 \%$ ), registando-se uma diferença significativa na sua distribuição em função do sexo ( $2=18.606 ; p=.005$ ). Os rapazes/homens apresentaram alterações de peso mais acentuadas, ou seja, em média mais $0.66( \pm 0.33) \mathrm{Kg}$. 
Por outro lado, observam-se igualmente diferenças entre raparigas e rapazes aquando da análise da distribuição dos valores do índice de massa corporal ( $p=.002)$. Os rapazes/ homens com o IMC mais elevado tenderam, por outro lado, a aumentar mais o seu peso, estando esse acréscimo associado à sua ocupação no momento $(\mathrm{p}=.008)$, com maior acréscimo de peso para os rapazes/ homens trabalhadores, muitos em teletrabalho.

Estes jovens, que nos seus tempos livres na infância nem todos aderiram a qualquer tipo de prática artística, física e desportiva, havendo maior adesão à prática física e desportiva (82.2\%), em meados de 2020 apresentavam valores muito baixos de adesão a uma prática de vida saudável, pois poucos realizavam atividade física intensa e regular (28.4\%).

Sobre a adesão à prática de atividade física registada por este grupo de adolescentes e jovens adultos, observamos que no momento da recolha de dados muitos ainda não praticavam qualquer AF (24.3\%). De acordo com os seus testemunhos, poucos tomaram medidas para realizar AF durante o confinamento e logo a seguir, adotando formas de atividade diferenciadas. Constata-se, pela análise da Figura 1, que 21.0\% realizou AF com monitorização de especialista e, dos que praticaram AF em casa, 27.0\% seguiu tutoriais apresentados por especialistas, enquanto 8.0\% fizeram atividade sem controlo ou monitorização e 19.0\% só praticaram pontualmente.

Figura 1. Adesão à prática de Atividade Física (AF) em período de confinamento, dados de 2020. (Fonte: Autoras)

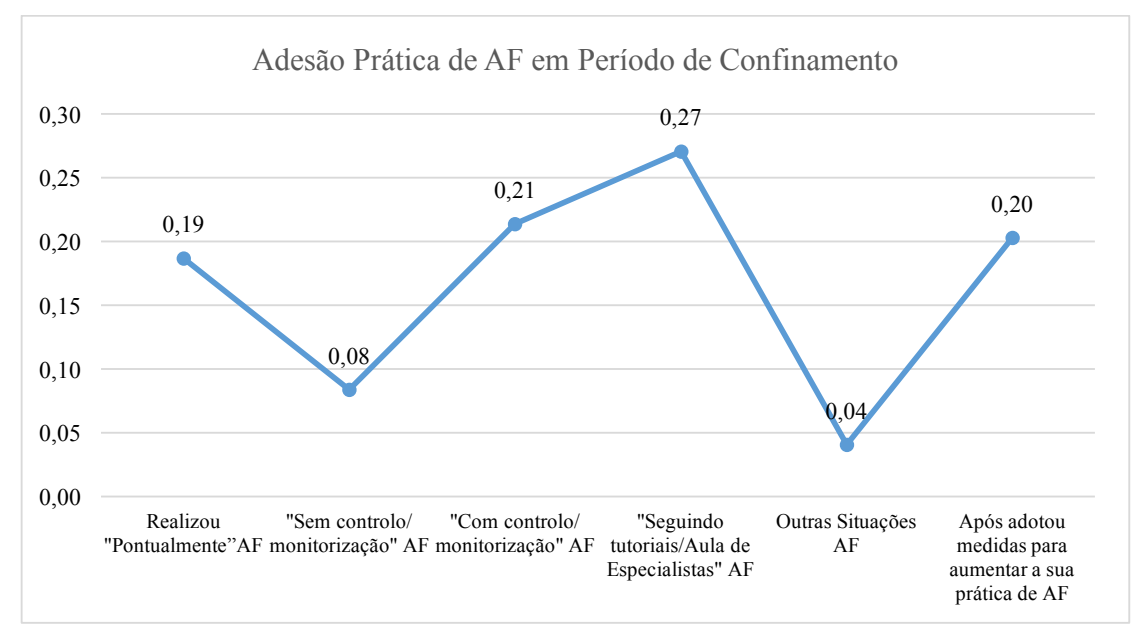

Por fim, tentámos perceber quais os fatores motivacionais que mais contribuíram para a adesão à prática de atividade física por este grupo de adolescentes e jovens adultos (Tabela 2).

Analisando de seguida os motivos que estes jovens apresentaram como mais relevantes para o seu envolvimento em práticas físicas e desportivas, estes foram vários e relacionam-se sobretudo com: 1.-. gosto pela prática física, artística e desportiva; 2. 0 o intuito de melhoria das capacidades; 3.. saúde e bem-estar e, por último, com menos expressão, e em 4..$^{\circ}$ lugar, pelo cuidado com o corpo. 
Tabela 2. Motivações com relevância na Adesão à Prática Atividade Física (\% e ), dados de 2020 (Fonte: Autoras)

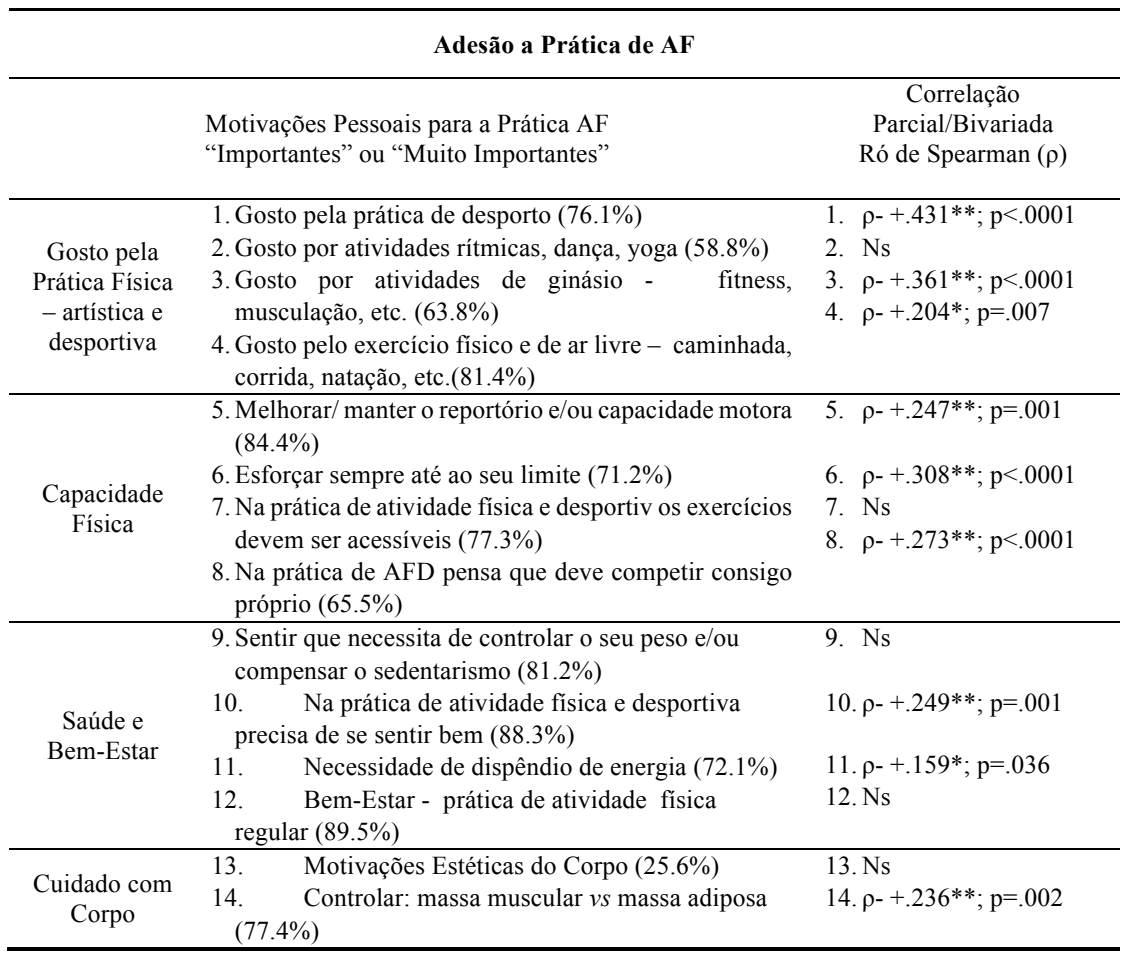

Quanto ao gosto pela prática, realça-se a importância dada ao gosto pelo exercício físico e de ar livre (81.4\%), motivo que aumentou ao longo do período de pandemia e onde se registou alguma relação com a idade, na medida em que obtivemos valores estatisticamente significativos para a Correlação de Spearman, que foi baixa, mas positiva ( =+.183; $p=.015$ ), ou seja, quanto mais velhos, maior adesão a prática. Também se encontraram diferenças entre aqueles que consideram menos e mais importante a prática de AF e a variação do fator peso, revelando assim a motivação e alteração de peso uma relação de dependência significativa indicada pelo teste de Qui-Quadrado ( $\mathrm{p}=.023)$. Salienta-se ainda a relevância dada ao gosto pela prática de desporto (76.1\%) e por atividades de ginásio (63.8\%), que reduziram em período de confinamento e evidenciaram uma correlação negativa e baixa, mas estatisticamente significativa com a habilitação académica ( = -.166; p=.027), estando provavelmente associado à prática de Educação Física em condições de Ensino à distância e a menor atividade para indivíduos com habilitações superiores, possivelmente muito ocupados com o (tele)trabalho não se predispondo a praticar AF.

Outros motivos aduzidos à adesão à AF foram melhorar e manter o reportório e/ou capacidade física e motora (84.4\%), esforçar sempre até ao seu limite (71.2\%) e pensar que deve competir consigo próprio (65.5\%), assim como, associado ao bem-estar e saúde, ao sentir-se bem (88.3\%) e a necessidade de dispêndio de energia (72.1\%).

Por fim, no cuidado com o corpo, as questões de estéticas revelaram-se associadas negativamente ao sexo ( =-489; p<.0001), havendo mais raparigas movidas por esta motivação, assim como se encontrou uma correlação negativa baixa, mas significativa, com a habilitação académica ( =-.171; p=.022), indicando que um nível aca- 
démico mais elevado se traduziu em mais baixa motivação para AF com vista ao cuidado com o corpo. Contudo, essa interação não se mantém com o nível de adesão à Atividade Física Regular. Aqui é, essencialmente, a necessidade de controlo da proporção massa muscular vs massa adiposa (77.4\% considera importante), onde 0 fator idade foi também relevante ( =+.225; $p=.003$ ), com os mais velhos a reconhecer mais benefícios da prática de AF.

\section{DISCUSSÃO E CONCLUSÕES}

Neste estudo registamos que a adesão a uma prática de atividade física regular não foi generalizada durante o confinamento imposto pela pandemia COVID-19 e encontramos prevalência de adolescentes e jovens com excesso de peso e/ou obesidade.

No período de confinamento houve alguma alteração de peso, com maior realce para 0 aumento de peso, situação que registou valores diferentes consoante o sexo dos indivíduos, o índice de massa corporal e a ocupação, alterações mais registadas nos rapazes/homens, nos indivíduos com excesso de peso/obesidade e nos jovens trabalhadores em teletrabalho.

A nível de adesão à pratica de AF regular no período considerado, em que muitos ginásios fecharam e muito tempo foi passado em casa, a variação apontou para um quinto (20\%) com maior adesão e outro quinto com menor adesão. Para Almeida e colegas (2021) o período de distanciamento social e de confinamento domiciliário necessário de adotar em período COVID-19 terá facilitado uma rotina mais sedentária, associada a ganho de peso. Consequentemente, também se associa a um possível aumento de risco cardiovascular bem como riscos acrescidos de complicações por infeção de COVID-19 (Dietz \& Santos-Burgoa, 2020).

Relativamente à prática de $\mathrm{AF}$ em casa predominou a prática de $\mathrm{AF}$ seguindo tutoriais, bem como aquela com algum controlo ou monitorização profissional, sendo às vezes pela própria escola na Educação Física Escolar, proporcionada à distância por videoconferência em que os alunos executavam os exercícios sob orientação dos professores da disciplina, estando em consonância com a opinião de Condessa (2015) sobre o papel dos profissionais de Educação Física intervirem eficazmente junto dos jovens e, mais recentemente, com Almeida et al (2021, p. 41) que enaltecem o papel dos profissionais de exercício físico, que conjuntamente com os profissionais de saúde deverão fomentar essas práticas, mesmo que em casa, visando reduzir os prejuízos ocasionados pelo distanciamento social.

Foram vários os motivos aduzidos ao nível de prática de AF regular, sendo os mais favoráveis à maior adesão o gosto pela atividade física, com a prática em ar livre; a necessidade de melhorar capacidade física e motora; seguindo-se a necessidade de se sentir bem no respeitante a saúde e bem-estar.

Por fim, o cuidado com o corpo foi menos mencionado, sendo o fator idade relevante para a importância de controlo da proporção da massa adiposa. As motivações de índole estética surgiram mais associadas às raparigas/mulheres, embora estas motivações não constituíssem um motivo ligado ao nível de adesão para a prática de AF regular.

Em suma, era de certo modo expectável que com o confinamento e a obrigatoriedade de permanecer em casa, obtivéssemos estes resultados. 0 teletrabalho e ensino a distância aumentaram o tempo de permanência em frente aos écrans, em posição sentada, praticamente imóvel, aumentando assim o sedentarismo e contribuindo para 0 aumento de peso. Contudo, acreditamos que alguns esforços individuais e preocupações com a condição corporal, bem como algumas motivações e 0 decorrer das aulas (obrigatórias) talvez tenham contribuído para uma percentagem de resultados positivos e minimizado os efeitos nefastos do confinamento para a saúde física e mental.

\section{FINANCIAMENTO}

Este trabalho foi financiado por Fundos Nacionais através da FCT - Fundação para a Ciência e a Tecnologia no âmbito do projeto do CIEC (Centro de Investigação em Estudos da Criança da Universidade do Minho) com a referência UIDB/00317/2020. 


\section{REFERÊNCIAS BIBLIOGRÁFICAS}

Almeida, M., Santos, C.G., Cruz, M.B., Fernandes, A.P.C., Isidorio, A.R., Júnior, M.L.O. (2021). Aspetos Associados à Prática de Atividade Física durante a Pandemia de COVID-19. In L.M.V. Souza (Org.). Educação para a Atividade Física e Saúde, pp. 34-43. Ponta Grossa - PR: Atena Editora. D0I: 10.22533/at.ed.7762109044.

Anastácio, Z. (2016). Self-esteem, Assertiveness and Resilience in Adolescents Institutionalized. Revista INFAD de Psicología, International Journal of Developmental and Educational Psychology, №1-Vol.1, 2016. ISSN: 0214-9877, pp. 315-322 (D0I: http://dx.doi.org/10.17060/ijodaep.2016.n1.v1.231)

An R. Projecting the impact of the coronavirus disease-2019 pandemic on childhood obesity in the United States: A microsimulation model. J Sport Health Sci. 2020 Jul;9(4):302-312. doi: 10.1016/j.jshs.2020.05.006. Epub 2020 May 23. PMID: 32454174; PMCID: PMC7250129.

Cardoso, C.S.; Condessa, I.C. \& Anastácio, Z.C. (2019). Hábitos de Vida Saudável no Pré-escolar e 1ํ Ciclo do Ensino Básico: a perceção de familiares e de educadores de um grupo de crianças. Revista INFAD de Psicología, International Journal of Developmental and Educational Psychology, 1(2), 203-214. Disponível, em http://www.infad.eu/RevistaINFAD/OJS/index.php/IJODAEP/article/view/1689

Cohen, L., Manion, L., \& Morrison, K. (2017). Research Methods in Education (8th ed.). Routledge. https://doi.org/10.4324/9781315456539

Condessa, I. \& Anastácio, Z. (2019). Estudo exploratório dos níveis de satisfação com a Imagem Corporal em Adolescentes - pensando no direito ao "bem-estar". Revista INFAD de Psicología, International Journal of Developmental and Educational Psychology, № 2, 2019. ISSN: 0214-9877. pp: 315-326 (D0I: https://doi.org/10.17060/ijodaep.2019.n2.v1.1701)

Condessa, M.I.C. (2015). A Problemática da Obesidade e da Atividade Física no Desenvolvimento Infantojuvenil: Professores (In)capazes? In E. Souza, J. Nascimento, E. Azevedo. \& B. Pereira. (Org.). Educação Física, Lazer e Saúde: interfaces ao desenvolvimento humano. Coleção Temas em Movimento. Volume 6., pp:277-298, Florianópolis: Editora da UDESC. ISBN: 978-85-8302-049-3. http://hdl.handle.net/10400.3/4065

Dietz W, Santos-Burgoa C. Obesity and its Implications for COVID-19 Mortality. Obesity (Silver Spring). 2020 Jun; 28(6):1005. doi: 10.1002/0by.22818. Epub 2020 Apr 18. PMID: 32237206.

Direção Geral da Saúde (2017). Programa Nacional para a Promoção da Atividade Física. Ministério da Saúde, Portugal. Acedido em janeiro, 8, em https://www.sns.gov.pt/wp-content/uploads/2017/10/DGS_PNPAF2017_V7.pdf

Direção-Geral da Saúde (2020). Programa Nacional para a Promoção da Atividade Física. Direção-Geral da Saúde. Acedido a 15 de abril de 2021. Disponível em: https://www.dgs.pt/programa-nacional-para-a-promocao-da-atvidade-fisica/ficheiros-externos-pnpaf/relat_pnpaf2020-pdf.aspx

Jakobsson, J., Malm, C., Furberg, M., Ekelund, U. \& Sevensson, M. (2020). Physical Activity During the Coronovirus (COVID-19) Pandemic Prevention of a Decline in Metabolic and Immunological Functions. Frontiers in Sports and Active Leaving. 2 (57). Doi: 10.3389/fsport.2020.00057.

Seabra, A. (2017). A Atividade Física em Crianças e Adolescentes. Um Comportamento decisivo para um estilo de vida saudável. Revista Factores de Risco, 44, abril/junho, 9- 20.

Silva, P., Graça, P., Mata, F., Arriaga, M.T. \& Silva, A.J. (2019). Estratégia Nacional para a Promoção da Atividade Física, da Saúde e do Bem-Estar I 2016-2025. Lisboa: Direção-Geral da Saúde (DGS).

World Health Organization (2020). FIFA launches \#ReachOut campaign for better mental health. Disponível em: https://www.who.int/news/item/02-08-2021-fifa-launches-reachout-campaign-for-better-mental-health

World Health Organization (2018). Global Action Plan on Physical Activity 2018-2030: More Active People for a Healthier World. Genebra. 